\title{
The odd chromatic index of almost all graphs*
}

\author{
Fábio Botler ${ }^{1}$, Lucas Colucci, and Yoshiharu Kohayakawa ${ }^{2}$ \\ ${ }^{1}$ Programa de Engenharia de Sistemas e Computação, COPPE, \\ Universidade Federal do Rio de Janeiro \\ Brazil \\ ${ }^{2}$ Departamento de Ciência da Computação \\ Instituto de Matemática e Estatística \\ Universidade de São Paulo \\ Brazil
}

\begin{abstract}
An odd coloring of a graph $G$ is an assignment of colors to the edges of $G$ in a way that each vertex is incident to either zero or an odd number of edges of each color. The minimum number of colors needed in such a coloring is called the odd chromatic index of $G$, and it is denoted by $\chi_{o}^{\prime}(G)$. This notion was introduced by Pyber, who showed that $\chi_{o}^{\prime}(G) \leq 4$ holds for every graph $G$. In this paper, we show that almost every graph on an even (resp. odd) number of vertices satisfies $\chi_{o}^{\prime}(G)=2\left(\right.$ resp. $\left.\chi_{o}^{\prime}(G)=3\right)$.
\end{abstract}

\section{Introduction}

The word graph in this paper refers to a graph without loops or multiple edges (i.e., a simple graph). A graph $G$ is called odd if all of its vertices have odd degree. An odd coloring of a graph $G$ is a collection of not necessarily spanning edge-disjoint odd subgraphs of $G$ that cover $E(G)$. In other words, an odd coloring of $G$ is a not necessarily proper edge-coloring of $G$ in which every vertex is incident to either zero or an odd number of edges of each color, i.e., of the same element of the collection. The odd chromatic index of $G$, denoted by $\chi_{o}^{\prime}(G)$, is the least number of subgraphs (equivalently, of colors) in an odd coloring of $G$. It is clear that $\chi_{o}^{\prime}(G)=0$ if and only if $G$ is empty; and $\chi_{o}^{\prime}(G)=1$ if and only if $G$ is an odd graph. The odd coloring problem was introduced by Pyber (1991), who proved that $\chi_{o}^{\prime}(G) \leq 4$ for every graph (see Figure 1).

A natural problem is to characterize the families of graphs with given odd chromatic index. In 2018, Kano, Katona, and Varga presented a polynomial time algorithm to decide whether a given multigraph has odd chromatic index 2. In 2006, Mátrai presented an infinite family of connected graphs $G$ with odd chromatic index 4, but a complete characterization of them seems to be difficult. We prove, on the other hand, that they are rare, in a sense that we will make clear below.

*This research has been partially supported by Coordenação de Aperfeiçoamento de Pessoal de Nível Superior - Brasil - CAPES - Finance Code 001. F. Botler is supported by CNPq (423395/2018-1), and by FAPERJ (211.305/2019). Y. Kohayakawa was partially supported by CNPq (311412/2018-1, 423833/20189) and FAPESP (2018/04876-1). The research that led to this paper started in WoPOCA 2019, which was financed by FAPESP (2015/11937-9) and CNPq (425340/2016-3, 423833/2018-9). FAPERJ is the Rio de Janeiro Research Foundation. FAPESP is the São Paulo Research Foundation. CNPq is the National Council for Scientific and Technological Development of Brazil. 

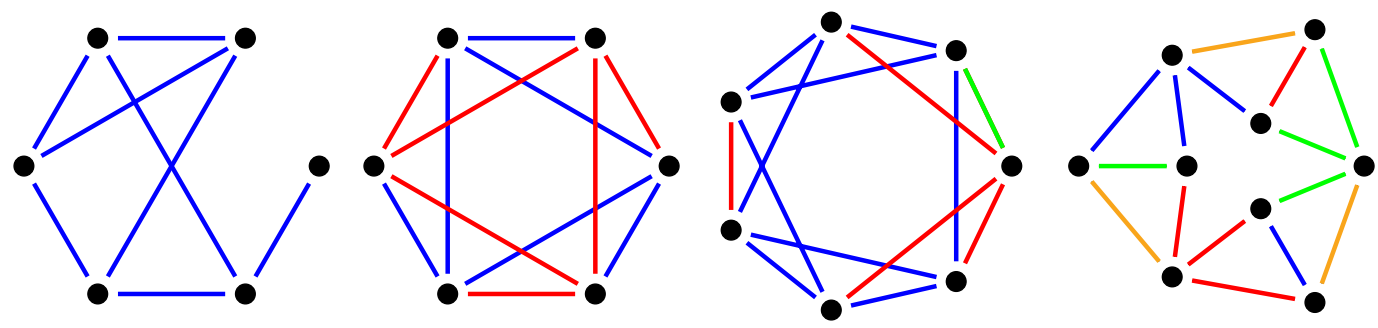

Figure 1. Examples of graphs with odd chromatic index equal to 1, 2, 3 and 4

The random graph $G(n, p)$ is the graph on $n$ vertices (or, to be precise, the probability distribution on the graphs on $n$ labeled vertices) where each edge is included independently and with probability $p$. In particular, $G(n, 1 / 2)$ is the distribution that assigns equal probability to each of the graphs on $n$ vertices. The main result of this paper says that if $n$ is even (resp. odd), the proportion of graphs on $n$ vertices and $\chi_{o}^{\prime}(G)=2$ (resp. $\chi_{o}^{\prime}(G)=3$ ) among the graphs with $n$ vertices tends to 1 when $n$ tends to infinity.

Theorem 1. There is a constant $C>0$ with the following property: if $p=p(n)<$ $1-1 / C$ and $p>C \sqrt{\frac{\log n}{n}}$, then, as $n \rightarrow \infty$, we have $\mathbb{P}\left(\chi_{o}^{\prime}(G(2 n, p))=2\right) \rightarrow 1$ and $\mathbb{P}\left(\chi_{o}^{\prime}(G(2 n+1, p))=3\right) \rightarrow 1$. In particular, by taking $p=1 / 2$, almost all graphs $G$ on an even (resp. odd) number of vertices satisfy $\chi_{o}^{\prime}(G)=2\left(\right.$ resp. $\left.\chi_{o}^{\prime}(G)=3\right)$.

This work is organized as follows. In Section 2, we present $S$-joins, which constitute a fundamental tool in the study of odd colorings; in Section 3, we sketch the proof of Theorem 1; and, in Section 4, we make some concluding remarks.

We thank the referees for their valuable comments and suggestions.

\section{2. $S$-joins}

Given a connected graph $G$ and a subset $S \subseteq V(G)$ with an even number of vertices, an $S$-join is a subgraph $H \subseteq G$ with the following property: for every $v \in V(H), d_{H}(v)$ is odd if and only if $v \in S$. Such a subgraph can be constructed by taking an arbitrary pairing of the vertices in $S$, taking (distinct) paths joining the vertices in each pair, and adding an edge to $H$ if and only if it is used in an odd number of the paths. Notice that the removal of the edges of a cycle of $H$ does not modify the parities of its vertices. Thus, any minimal $S$-join is a forest.

Since the odd chromatic index of a graph is equal to the maximum of the odd chromatic indices of its connected components, we assume, in what follows, that the graphs we are dealing with are connected, even without stating it explicitly. Also, for a graph $G$, we denote by $G_{e}$ and $G_{o}$ the subgraphs of $G$ induced by the vertices of even and odd degrees, respectively. An Eulerian graph is a connected graph in which every vertex has even degree, i.e., a connected graph $G$ for which $G_{e}=G$.

We now present some known results on the odd chromatic index obtained by using $S$-joins.

Proposition 1 (Pyber 1991). If $G$ is an Eulerian graph with $|V(G)|$ even, then $\chi_{o}^{\prime}(G)=2$. Proof. Let $H$ be an $S$-join of $G$, with $S=V(G)$. Note that both $H$ and $G \backslash E(H)$ are odd graphs. Therefore $\{H, G \backslash E(H)\}$ is an odd coloring of $G$. On the other hand, $\chi_{o}^{\prime}(G)>1$ since $G$ is not odd itself. 
Proposition 1 is tight in the sense that if $G$ has an even number of vertices, but is not Eulerian (resp. Eulerian, but has an odd number of vertices), then two colors may not suffice: the graph obtained by joining two vertices by three internally disjoint paths, two of length two and one of length three (resp. an odd cycle) has odd chromatic index 3.

Proposition 2 (Pyber 1991). If $G$ is a graph such that $|V(G)|$ is even, then $\chi_{o}^{\prime}(G) \leq 3$.

Proof. A forest can be odd colored with two colors (applying a greedy coloring in a breadth-first ordering of the edges in each tree component). Now, let $G$ be a graph on an even number of vertices. In this case, $\left|V\left(G_{e}\right)\right|$ is even. Let $H$ be a minimal $S$-join of $G$ with $S=V\left(G_{e}\right)$. Since $H$ is a forest, we have $\chi_{o}^{\prime}(H) \leq 2$. Moreover, since $H$ is a $V\left(G_{e}\right)$-join, $G \backslash E(H)$ is an odd graph. This concludes the proof.

\section{The odd chromatic index of almost all graphs}

Note that the proof of Proposition 1 uses the fact that a $V\left(G_{e}\right)$-join is an odd graph. This property also holds if each connected component of $G_{e}$ contains an even number of vertices. Indeed, let $S_{1}, \ldots, S_{k}$ be the components of $G_{e}$, and note that a $V\left(S_{i}\right)$-join $J_{i}$ of $S_{i}$ is an odd subgraph of $S_{i}$. Thus, $G \backslash\left(\bigcup_{i=1}^{k} E\left(J_{i}\right)\right)$ is also an odd graph. Therefore $\chi_{o}^{\prime}(G)=2$ unless $G$ is an odd graph. In particular, this implies that if a graph $G$ has an even number of vertices (so $\left|V\left(G_{e}\right)\right|$ is even as well) and $G_{e}$ is connected, then $\chi_{o}^{\prime}(G) \leq 2$.

On the other hand, if $G$ has an odd number of vertices and $G_{e}$ and $G_{o}$ are connected and $G_{o}$ is nonempty, we must have $\chi_{o}^{\prime}(G) \geq 3$. Indeed, if $v$ is a vertex of odd degree, then either one or three colors are used on the edges incident to $v$. Thus, if $\chi_{o}^{\prime}(G) \leq 2$, then every vertex of odd degree has all the edges incident to it with the same color. This implies, as $G_{o}$ is connected, that all the edges incident to $V\left(G_{o}\right)$ are colored with a single color, say, 1 . Thus, the graph spanned by the edges of color 2 is an odd spanning subgraph of $G_{e}$. This is a contradiction, since $\left|V\left(G_{e}\right)\right|$ is odd.

Theorem 1 is a consequence of Lemma 1, which says that for almost all graphs $G$, the graphs $G_{e}$ and $G_{o}$ are connected. Indeed, if $|V(G)|$ is even, $\chi_{o}^{\prime}(G)=2$ follows directly from the fact that $G_{e}$ is connected. On the other hand, suppose that $|V(G)|=n$ is odd. Fix a vertex $v$ in $G$. With probability that tends to 1 as $n \rightarrow \infty, v$ has a neighbor $u$ of even degree and $\chi_{o}^{\prime}(G-v)=2$. We extend a 2-coloring of $G-v$ to a 3-coloring of $G$ in the following way: if $d(v)$ is odd, color all the edges incident to $v$ with a new color; if $d(v)$ is even, color all the edges incident to $v$ but $u v$ with a new color and color $u v$ with the color used in the coloring of $G-v$ which does not appear in the edges incident to $u$ in this graph (such a color exists since $d_{G-v}(u)$ is odd). The result now follows from the bound $\chi_{o}^{\prime}(G) \geq 3$ from the previous paragraph.

Lemma 1. There is a constant $C>0$ with the following property: if $p=p(n)<1-1 / C$ and $p>C \sqrt{\frac{\log n}{n}}$, then $\mathbb{P}\left(G(n, p)_{e}\right.$ and $G(n, p)_{o}$ are connected graphs $) \rightarrow 1$ as $n \rightarrow \infty$.

Sketch of the proof. The idea of the proof is inspired by a lemma of Korándi, Krivelevich and Sudakov [Korándi et al. 2015].

First, note that the probability that the Binomial variable $\operatorname{Bin}(m, p)$ is odd (resp. even) is equal to $\frac{1-(1-2 p)^{m}}{2}$ (resp. $\frac{1+(1-2 p)^{m}}{2}$ ). We use the following version of the Chernoff bound (see, e.g., [Alon and Spencer 2004]): Let $X_{1}, \ldots, X_{n}$ be independent Bernoulli 
variables with $\mathbb{P}\left(X_{i}=1\right)=p_{i}$. Let $X=\sum_{i=1}^{n} X_{i}$ and $\mu=\mathbb{E}(X)=\sum_{i=1}^{n} p_{i}$. Then, for any $\varepsilon>0$, there is $c_{\varepsilon}>0$ such that $\mathbb{P}(|X-\mu|>\varepsilon \mu) \leq 2 e^{-c_{\varepsilon} \mu}$.

In what follows, we prove the stronger statement that the probability that for every $u$ and $v$ in $V(G)$, there is a vertex of even degree $x_{e}$ and a vertex of odd degree $x_{o}$ adjacent to $u$ and $v$ tends to 1 . It is enough to prove the statement for even degree vertices, as the proof for odd degrees vertices is similar. Consider two distinct vertices $u$ and $v$ in $V(G)$. We estimate the probability that all the common neighbors of $u$ and $v$ have odd degree in the following way: we fix a set $A \subseteq V(G)$ not containing $u$ and $v$ such that $|A|=n-\left\lfloor n^{2 / 3}\right\rfloor$ and put $B=V(G)-A-\{u, v\}$. Let $X$ be the random variable that counts the number of vertices in $A$ that are joined to both $u$ and $v$ and have even degree. We generate $G(n, p)$ in two steps: first, we expose the edges inside $A$ and $B$. Conditioning on any collection of edges spanned by $A$ and $B, X$ is the sum of $|A|$ independent Bernoulli variables (corresponding to each vertex of $A$ ) with parameter either $\bar{p}_{1}=p^{2} \cdot \frac{1+(1-2 p)^{|B|}}{2}$ or $\bar{p}_{2}=p^{2} \cdot \frac{1-(1-2 p)^{|B|}}{2}$. In any case, $\mu=\mathbb{E}(X) \sim n p^{2} / 2$. Applying the Chernoff bound to the variable $X$ with $\varepsilon=1$, we get that $\mathbb{P}(|X-\mu|>\mu) \leq 2 e^{-c \mu}$, for some $c>0$. Thus, $\mathbb{P}(X=0) \leq 2 e^{-c \mu}$. By the union bound, we have that the probability that some pair of vertices in $G(n, p)$ does not have an even degree common neighbor is at most $\left(\begin{array}{l}n \\ 2\end{array}\right) 2 e^{-c \mu}<e^{2 \log n-c \mu} \rightarrow 0$ if $C>2 c^{-1 / 2}$.

\section{Concluding remarks}

We established the odd chromatic index of almost all graphs, and proved that it depends only on the parity of the number of vertices of the graph. It might also be interesting to prove a result similar to Theorem 1 for multigraphs, for which the odd chromatic index can go up to 6 [Lužar et al. 2014, Petruševski 2018]. In another direction, we may consider decompositions in graphs in which every vertex has degree 1 modulo $k$. In this case, the best known upper bound to the modulo $k$ chromatic index is $5 k^{2} \log k$ [Scott 1997].

\section{References}

Alon, N. and Spencer, J. H. (2004). The probabilistic method. John Wiley \& Sons.

Kano, M., Katona, G. Y., and Varga, K. (2018). Decomposition of a graph into two disjoint odd subgraphs. Graphs and Combinatorics, 34(6):1581-1588.

Korándi, D., Krivelevich, M., and Sudakov, B. (2015). Decomposing random graphs into few cycles and edges. Combinatorics, Probability and Computing, 24(6):857-872.

Lužar, B., Petruševski, M., and Škrekovski, R. (2014). Odd edge coloring of graphs. Ars Mathematica Contemporanea, 9(2):267-277.

Mátrai, T. (2006). Covering the edges of a graph by three odd subgraphs. Journal of Graph Theory, 53(1):77-82.

Petruševski, M. (2018). Odd 4-edge-colorability of graphs. Journal of Graph Theory, 87(4):460-474.

Pyber, L. (1991). Covering the edges of a graph by .... In Sets, Graphs and Numbers, Colloquia Mathematica Societatis János Bolyai, volume 60, pages 583-610.

Scott, A. D. (1997). On graph decompositions modulo k. Discrete Mathematics, 175(13):289-291. 